\title{
Where is the catheter? an unexpected complication from a paravertebral catheter placement
}

\begin{abstract}
Background: Placement of a thoracic paravertebral nerve block catheter (PVBC) is a safe and effective method for providing postoperative analgesia for pediatric patients undergoing thoracotomy. The PVBC can be misplaced despite accurate needle placement.

Case presentation: An 8 year old girl with a history of right lower extremity osteosarcoma and a right lung nodule underwent thoracotomy and wedge resection of the right lung. A T7 paravertebral nerve block catheter (PVBC) was placed under ultrasound guidance for postoperative pain relief. The patient reported satisfactory analgesia at thoracic incision without side effects throughout postoperative anesthesia care unit stay and postoperative day 1 (POD1). On POD2, the patient reported a droopy left eyelid and disclosed blurry vision since POD1. The physical exam revealed left partial Horner's syndrome and numbness along the left T5-7 dermatome. The PVBC insertion depth was unchanged. The ptosis and miosis resolved completely one hour after stopping the ropivacaine infusion. The infusion was restarted after the catheter was pulled back by $2 \mathrm{~cm}$ and bloused with 5 $\mathrm{ml}$ of ropivacaine $0.2 \%$. Patient reported that her pain was relieved with no recurrence of her symptoms.
\end{abstract}

Discussion: This is the first case report of a patient who developed contralateral Horner's syndrome from the placement of a PVBC. The PVBC can reach the contralateral paravertebral space via inter vertebral foramina or prevertebral space.

Conclusion: The paravertebral catheter depth in children may need to be individualized.
Volume I Issue 5 - 2014

\author{
Mihaela Visoiu,' Shente Steven Hsu² \\ 'Department of Anesthesiology, University of Pittsburgh Medical \\ Centre, USA \\ ${ }^{2}$ Department of Anesthesiology, Shadyside Hospital of UPMC, \\ USA
}

\author{
Correspondence: Mihaela Visoiu, Department of \\ Anesthesiology, Acute Interventional Perioperative Pediatric \\ Postoperative Pain Management, Children's Hospital of \\ Pittsburgh of Children's Hospital Drive, 440I Penn Avenue, \\ Pittsburgh, PA 15224, USA, Tel 4I2-692-5260, \\ Email visoium@upmc.edu
}

Received: October 31, 2014 | Published: November 12, 2014

Keywords: Horner's syndrome; Child; Local anesthetic; Regional; Ultrasound

Abbervations: PVBC: Paravertebral Nerve Block Catheter; POD: Postoperative Day; CT: Computed Tomography

\section{Introduction}

Placement of a thoracic paravertebral nerve block catheter (PVBC) is a safe and effective method for providing postoperative analgesia for pediatric patients undergoing thoracotomy. ${ }^{1}$ Few cadaveric studies showed that paravertebral catheters can be misplaced despite the correct placement of needle, but there is no case report of a catheter misplaced into the contra lateral paravertebral space. ${ }^{2}$ We report a case of a PVBC insertion which resulted in a contra lateral Horner's syndrome.

\section{Case report}

A $24.3 \mathrm{~kg} 8$ year old girl with a history of right lower extremity osteosarcoma and a right lung nodule underwent thoracotomy and wedge resection of the right lung. Postoperative pain was controlled by a right PVBC and multimodal analgesia. Following surgery, while the patient remained under general anesthesia and in the lateral position, the T7 paravertebral space was identified using a high frequency ultrasound probe (Sonosite, $6-15 \mathrm{MHz}, 5 \mathrm{~cm}$ linear probe) connected to a Sonosite S-Nerve machine (Bothnell, WA) as described by Boretsky et al. ${ }^{1}$ Under direct ultrasound visualization, a $9 \mathrm{~cm}, 18$ gauge Tuohy needle (Pajunk Tuohy Sono, Geisingen, Germany) was inserted in-plane, from lateral to medial, in a slightly oblique orientation. After confirming correct needle placement by anterior displacement of pleura, $10 \mathrm{ml}$ of ropivacaine $0.2 \%$ was administered. A 20 gauge catheter (Perifix Epidural Catheter, B. Braun Medical Inc. Bethlehem, PA) was inserted $3.5 \mathrm{~cm}$ beyond the needle tip, $8 \mathrm{~cm}$ at the skin level and an infusion of $5 \mathrm{ml} / \mathrm{hr}$ of $0.2 \%$ ropivacaine was started. The patient reported satisfactory analgesia at thoracic incision without side effects throughout postoperative anesthesia care unit stay and postoperative day 1 (POD1).

On POD2, the patient reported a droopy left eyelid and disclosed blurry vision since POD1. The neurology service ordered a chest computed tomography (CT) to rule out a vascular dissection and/ or brain metastasis. The patient reported no noticeable change in thoracic pain severity. The physical exam revealed left partial Horner's syndrome and numbness along the left T5-7 dermatome. The PVBC insertion depth was unchanged. The ptosis and miosis resolved completely one hour after stopping the ropivacaine infusion. The CT was cancelled. The patient reported increased pain over the surgical side and requested her paravertebral infusion be restarted. The infusion was restarted after the catheter was pulled back by $2 \mathrm{~cm}$ and bolused with $5 \mathrm{ml}$ of ropivacaine $0.2 \%$. Patient reported that her pain was relieved with no recurrence of her symptoms. The PVBC was removed on POD3.

\section{Discussion}

Horner's syndrome is a rare complication from paravertebral nerve blocks. ${ }^{3}$ Contralateral occurrence has not been previously reported. A possible cause could be the local anesthetic spreading toward the stellate ganglion during initial paravertebral injection, or through an existing PVBC. Local anesthetic injected into the paravertebral space can distribute into the epidural space or the contralateral paravertebral space. ${ }^{4}$ With a properly placed catheter, the ongoing infusion could track toward the left paravertebral space; however, a higher volume and pressure would be necessary to reach the contralateral stellate 
ganglion. This would not adequately explain the sensory blockade only along left T5-7. The PVBC can be misplaced despite accurate needle placement. ${ }^{2}$ In our case, we considered the possibilities of contralateral placement and the less likely left subdural or epidural placement.

The endothoracic fascia separates paravertebral space into anterior and posterior compartments. The spinal nerves are located in the posterior compartment, whereas the sympathetic ganglia are located in the anterior compartment. ${ }^{5}$ Local anesthetic spread in the paravertebral space is distributed mainly caudally and over only a few dermatomes. ${ }^{4}$ However, the presence of endothoracic fascia may affect the spread of local anesthetic. Local anesthetic infused anterior to the fascia meets more resistance and has a more longitudinal pattern. A small amount can spread over multiple segments, reaching the stellate ganglion.

Our patient did not develop Horner's syndrome after initial paravertebral injection. The initial needle position and ropivacaine may have been posterior to the endothoracic fascia. This fascia is very thin in dissected cadavers and can be perforated by the catheter. ${ }^{2}$ The PVBC can reach the contralateral paravertebral space the intervertebral foramina (Figure 1) or prevertebral space (Figure 2). In our case, Horner's syndrome developed after an appropriate infusion rate. We believe the catheter traveled anterior to the endothoracic fascia, into the anterior contralateral paravertebral space.

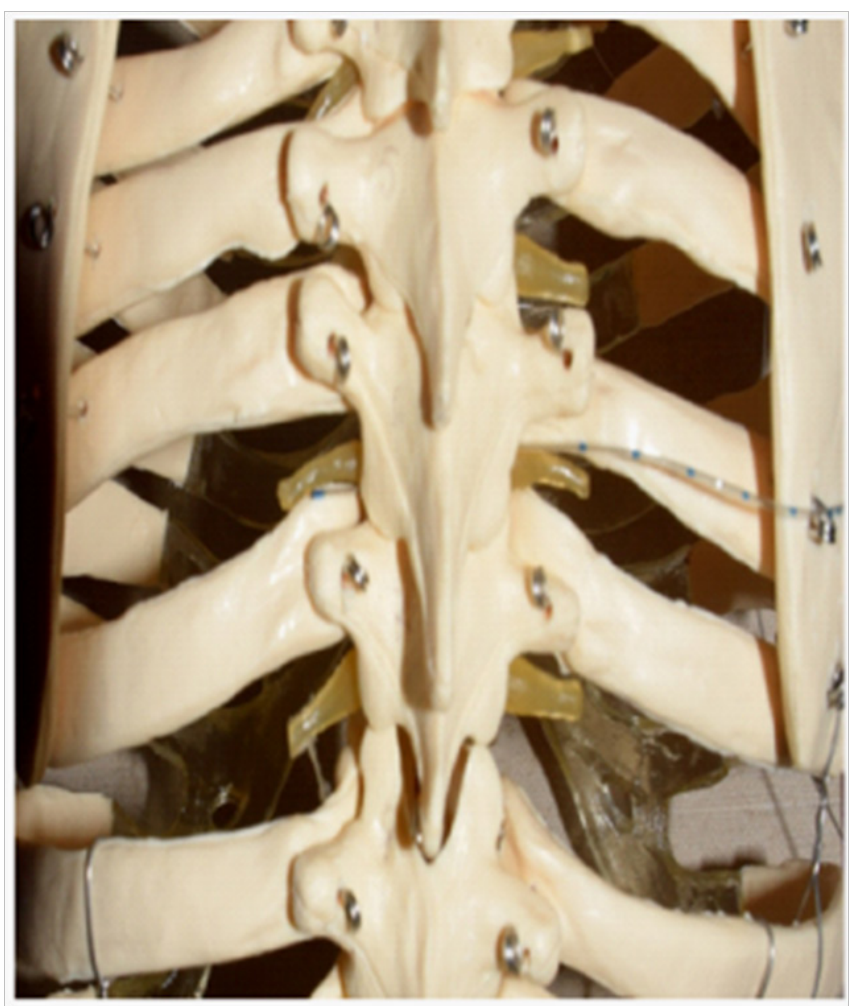

Figure I Paravertebral catheter reached contralateral paravertebral space via intervertebral for amina.

Despite the contralateral PVBC placement, our patient reported pain relief at the surgical site and increased pain upon stopping the infusion. The catheter has orifices located along the first $1.5 \mathrm{~cm}$ of its end. The distal orifice could have been located anterior to the endothoracic fascia on the left, with the proximal orifice close to the paravertebral space on the right.

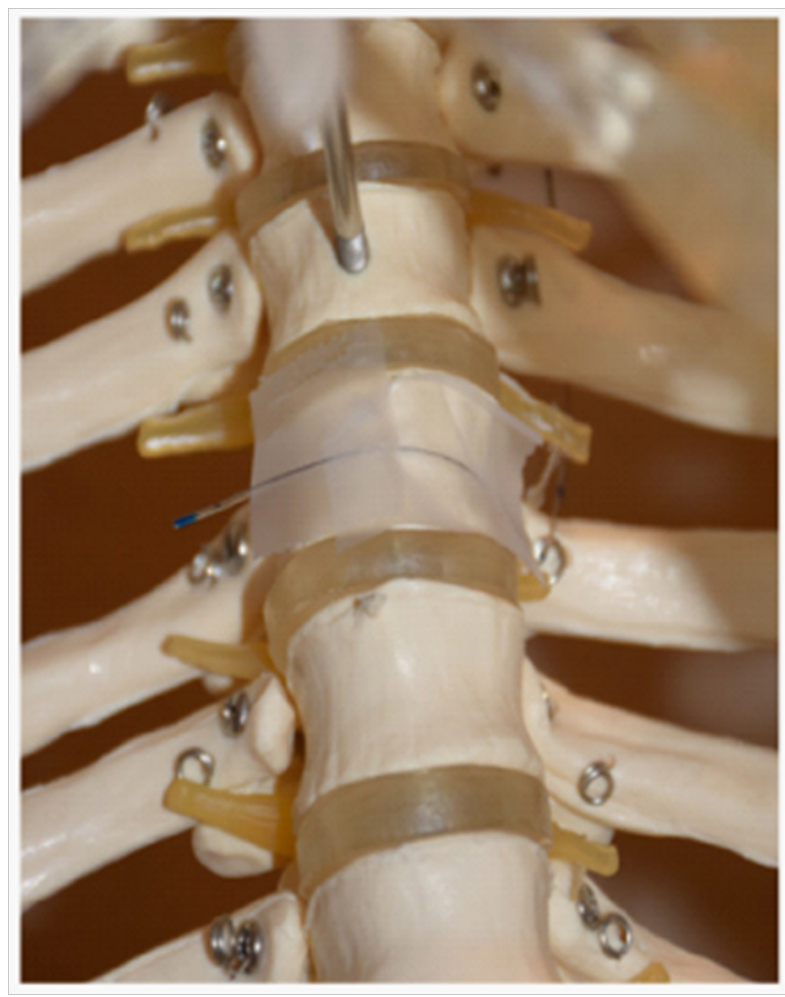

Figure 2 Prevertebral space.

\section{Conclusion}

In summary, this case of contralateral Horner's syndrome may be attributed to sympathetic dennervation caused by the paravertebral spread of ropivacaine to the left stellate ganglion, because the PVBC tip is able to reach the anterior compartment of the contralateral paravertebral space. PVBC placement in children will continue to be a significant challenge and catheter depth may need to be individualized.

\section{References}

1. Boretsky K, Visoiu M, Bigeleisen P. Ultrasound-guided approach to the paravertebral space for catheter insertion in infants and children. Pediatr Anesth. 2013;23(12):1193-1198.

2. Luyet C, Herrmann G, Ross S, et al. Ultrasound-guided thoracic paravertebral puncture and placement of catheters in human cadavers: where do catheters go? Br J Anaesth. 2011;106(2):246-254.

3. Renes SH, Bruhn J, Gielen MJ, Scheffer GJ, van Geffen GJ (2010) Inplane ultrasound-guided thoracic paravertebral block: a preliminary report of 36 cases with radiologic confirmation of catheter position. Reg Anesth Pain Med. 2010;35(2): 212-216.

4. Marhofer D, Marhofer P, Kettner SC, et al. Magnetic resonance imaging analysis of the spread of local anesthetic solution after ultrasoundguided lateral thoracic paravertebral blockade: a volunteer study. Anesthesiology. 2013;118(5): 1106-1112.

5. Stopar Pintaric T, Veranic P, Hadzic A, et al. Electron-microscopic imaging of endothoracic fascia in the thoracic paravertebral space in rats. Reg Anesth Pain Med. 2012;37(2): 215-218. 